\title{
Video Game (Film-)Essays: Der (etwas andere) Einsatz von Computerspielen zur Unterstützung von Lernprozessen
}

Ralf Biermann

\begin{abstract}
Unter dem Terminus "Video Game Essay» wird eine Form der filmischen Auseinandersetzung mit Computerspielen verstanden, bei der das Bildschirmgeschehen des Spiels die Ausgangslage für eine visuell orientierte Darstellungsform und Themenbearbeitung bildet. Diese hat - ähnlich wie die «klassische» Filmarbeit-die Förderung von Medienkompetenz und Selbstkompetenz zum Ziel. Der veränderte Produktionsprozess ist mit dem von Machinima-Filmen vergleichbar. Als Beispiele für mögliche Einsatzgebiete für diese besondere Form der Auseinandersetzung mit Digital Games werden in diesem Artikel die ausserschulische Medienarbeit, die Schul- und Hochschulausbildung genauer betrachtet.
\end{abstract}

Medienprojekte mit Computerspielen sind neben den klassischen Arbeitsfeldern (Video, Radio) der medienpädagogischen Praxisarbeit ein eher randständiges Thema. Dabei haben die Spiele in den letzten 15 Jahren eine zunehmende Bedeutung in den Kinder- und Jugendkulturen erlangt. Bei Kindern ist das Spielen «die am häufigsten ausgeübte Tätigkeit am Computer. Dabei beschäftigen sich ein Fünftel der kindlichen Spielnutzer jeden bzw. fast jeden Tag alleine oder gemeinsam mit anderen mit Computerspielen» (MPFS 2007, S. 33). Die meisten Spiele erhalten die Kinder von den Eltern und nur zu einem geringeren Teil von Freunden. Dabei erfolgt die Anschaffung der Spiele meist durch die Eltern (55 Prozent) und 30 Prozent der Kinder gaben an, sich Spiele selbst aussuchen zu dürfen. Nur bei zehn Prozent entscheiden ausschliesslich die Eltern, was gespielt werden darf (vgl. MPFS 2007, S. 34-35). Trotz des durchschnittlichen Besitzes von 13 Spielen pro Kind (vgl. MPFS 2007, S. 33) gibt es einen durchaus hohen Anteil von Kindern (27 Prozent), die gar nicht am Computer spielen (vgl. MPFS 2007, S. 37).

Bei den Jugendlichen ist ein ähnliches Bild zu verzeichnen: «So zählen 37 Prozent zu den intensiven Spielern (täglich/mehrmals pro Woche), weitere 15 Prozent spielen einmal pro Woche bzw. einmal in 14 Tagen. Ein Fünftel kann als eher sporadische Spieler beschrieben werden (einmal im Monat oder seltener) und immerhin 28 Prozent spielen überhaupt nicht» (MPFS 2006, S. 36).

An den Zahlen wird deutlich, welche Bedeutung Computerspiele in den Kinderund Jugendkulturen mittlerweile haben. Zwar ist die Relevanz unterschiedlich aus- 
geprägt (bei Vielspielern dürfte sie weitaus grösser sein als bei den Nichtspielern), allgemein kann man sie als ein wichtiges Element kindlicher und jugendlicher Lebenswelten einstufen. Aus diesem Grund bietet es sich geradezu an, auf die Spielerfahrungen in der aktiven Medienarbeit und in anderen Kontexten (Schule und Ausbildung von Studierenden) zurückzugreifen und bisherige Nutzungsmuster und Bewertungen zu reflektieren.

Während die Erstellung von Filmen, Radiobeiträgen und multimedialen Selbsterzeugnissen (z. B. im Kontext von Medienbiografien bei Kommer/Biermann 2007) als fest etabliert beschrieben werden kann, stehen Projekte mit Computerspielen eher selten im Fokus medienpädagogischer Praxisarbeit. ${ }^{1}$ Dies dürfte mitunter auf die mit Computerspielen verbundenen negativen Assoziationen in der Öffentlichkeit zurückzuführen sein. Insbesondere mit den Amokläufen in Erfurt und Emsdetten wurden lautstark Forderungen gestellt, für «Killerspiele» ein Produktionsverbot zu verhängen. Dies klang insofern nachvollziehbar, da man in den Spielen (jedoch ohne jegliche Beweisführung) einen wesentlichen Faktor für diese Taten sah.

Als Reaktion auf diese Amokläufe standen vor allem gewalthaltige Spiele in der Diskussion, die ohnehin erst ab 18 Jahren freigegeben sind. Betrachtet man hierzu die Angaben des Bundesverbandes Interaktive Unterhaltungssoftware e. V. in den vorgestellten Marktzahlen für das Jahr 2007 (vgl. Bundesverband Interaktive Unterhaltungssoftware e. V. 2007, S. 9), so wird deutlich, dass Spiele ohne Jugendfreigabe (also ab 18 Jahren) lediglich einen Marktanteil von sechs Prozent haben. Spiele ab 16 Jahren haben einen Anteil von zehn Prozent, ab 12 Jahren 23 Prozent, ab 6 Jahren 13 Prozent und ganze 47 Prozent erhalten eine Kennzeichnung ohne Altersbeschränkung. Über die Jahre hinweg ergeben sich nur kleinere Schwankungen, sodass hier von relativ stabilen Zahlen über den Verlauf der letzten Jahre gesprochen werden kann und ein Grossteil der Software durchaus für Projekte mit Kindern und Jugendlichen genutzt werden könnte. Die Diskussion über Gefährdungspotentiale betrifft lediglich einen kleinen Teil der sich auf dem Markt befindlichen Spiele. Trotzdem bezieht sich der Grossteil der öffentlichen Diskussion auf diese.

Kompetenzfördernde Potentiale von Computerspielen (vgl. Kraam 2004 sowie Gebel et al. 2005) werden in der Regel nur am Rande oder überhaupt nicht berücksichtigt. Dabei können Computerspiele in vielfältiger Weise für Lehr- und Lernzwecke eingesetzt werden (vgl. Fromme/Meder 2001), was sich auch in der vermehrten Verwendung des Begriffs Serious Games verdeutlicht, um den «ernsthaften» lernorientierten Charakter dieser Sparte aufzuzeigen (vgl. Egenfeldt-Nielsen/Smith/ Tosca, S. 205-206). Doch auch bei den Entertainmenttiteln finden sich ausreichend Potentiale zur Unterstützung formeller wie informeller Lernarrangements. Mit

Betrachtet man hierzu Literatur, in der Projekte gesammelt und dargestellt werden, so sind Computerspiele im Verhältnis zur Relevanz in der Lebenswelt von Kindern und Jugendlichen in Projekten unterrepräsentiert. 
dem Konzept der Video Game Essays - ursprünglich gedacht für die Analyse von Computerspielen - werden in diesem Artikel drei Möglichkeiten vorgestellt, das Medium Computerspiel in methodischer Anlehnung an die Kriterien der aktiven Medienarbeit (vgl. Schell 2003, S. 51ff) mit unterschiedlichen Zielgruppen einzusetzen. Dazu ist es zunächst notwendig, den anfänglichen Einsatzzweck und Gegenstand der Video Game Essays zu erläutern, um grundlegende Aspekte deutlich zu machen. Daran anschliessend werden die Möglichkeiten des Einsatzes in Lernprozessen der ausserschulischen Medienarbeit, der Hochschule und der Schule aufgezeigt. Es geht dabei nicht primär um Lerneffekte, die mit der Tätigkeit des Spielens zusammen hängen und durch die Wissen vermittelt werden soll, sondern vielmehr um reflexive Prozesse, die durch eine spezifische Auseinandersetzung mit dem Computerspiel initiiert werden. Diese reflexiven Prozesse sind auf der Metaebene angesiedelt und fragen nach Sinnzusammenhängen, in denen Computerspiele zur individuellen Weiterentwicklung (z. B. Bildungsaspekte im Sinne einer strukturalen Medienbildung wie bei Marotzki und Jörissen 2008 beschrieben) beitragen können.

\section{Video Game Essays als Analyseformat für Computerspiele}

Die Ursprungsidee, Filmessays über Computerspiele zu produzieren, basierte auf der Suche nach einer alternativen Analyseform zur textbasierten Beschreibung. Zu den ersten Analyseformen von Computerspielen im erziehungswissenschaftlichen Bereich kann die Vorgehensweise von Fritz (1995 und 1997) mit einer Verortung auf einer «Landkarte» zurückgeführt werden. Diese ist charakterisiert durch die Extrempole Action, Denken und Geschichten. Anhand dieser Kriterien werden die Spiele eingeordnet und beschrieben:

«So legen wir für die «Landkarte der Bildschirmspiele» drei Ausdehnungsrichtungen durch drei wesentliche Spielelemente zugrunde: «Denken〉, ‘Action〉 und «Geschichten` [...]. Je nachdem, wie stark ein einzelnes Spiel durch diese drei Elemente (wie ein Magnet) ‘angezogen` wird, desto deutlicher wird das Spiel in Richtung auf dieses Element auf der 〈Landkarte» placiert (sic)» (Fritz 1997, S. 87).

Es leuchtet ein, dass das nun nahezu 15 Jahre alte und nicht weiterentwickelte Analyseformat nicht mehr auf die aktuellen Computerspiele und deren enormen Entwicklungen bezogen werden kann. Fritz selbst schlägt aus diesem Grund vor, von einer Typologie zu einer Genealogie zu wechseln: "Eine adäquate Bestimmung und Einschätzung der Spielmuster hängt von ihrer Genealogie ab, d. h. von der Frage der historischen Entwicklung der Computerspiele in Hinblick auf ihre Merkmale und Elemente» (Fritz 1997, S. 97).

Auch andere Fachdisziplinen, z. B. die Psychologie, versuchten eine Annäherung an den Gegenstand Computer. Die Kriterien im Diskurs der Psychologen fallen sehr ähnlich zu denen der Pädagogen aus: Politischer Extremismus, Aggressivität, Kooperation, soziale Isolierung durch Zeitdruck, Faszination, Kognition, Lernen, Kre- 
ativität, Ästhetik, geschlechtsspezifische Problematik, Zielsetzung, Motorik, technische Entwicklung und technische Standards (vgl. Löschenkohl/Bleyer 1995, S. 36-55). Auch hier stehen mögliche Gefährdungspotentiale und eine Problemsicht nicht aussen vor, zurzeit dominieren diese mit der Computerspielsucht sogar den psychologischen Diskurs.

Mit dem Aufkommen der Computer Game Studies um 2001 (vgl. Aarseth 2001) nahm das Interesse an den digitalen Spielen zu. Fachspezifische Fragen wurden an das Medium herangetragen und immer öfter kam es zu interdisziplinären Betrachtungen (vgl. hierzu auch den Sammelband Neitzel/Bopp/Nohr 2005). Die Zunahme an Publikationen (on- wie offline) zeigen vor allem eines: Es findet eine Abkehr von problemzentrierten Sichtweisen hin zu der Frage statt, wie man das Medium Computerspiel beschreiben und in seiner Gesamtbedeutung begreifen kann.

Betrachtet man die wissenschaftlichen Beschreibungen und Analysen der Spiele, werden diese in schriftlicher Form fixiert und gelegentlich mit Screenshots - also Standbildern eines sehr lebendigen Mediums - unterfüttert (vgl. exemplarisch Neitzel/Bopp/Nohr 2005). Natürlich liegt dies auch in der typischen Verbreitung wissenschaftlicher Schriften über Bücher und Zeitschriften begründet. Die Problematik bei einem visuell orientierten Medium wie dem Computerspiel besteht darin, die (bewegte) Bildinformation in ein diskursives Muster zu transformieren, also von einem Informationskanal in einen anderen zu übersetzen². Hier setzen die Video Game Essays als filmische Form der Analyse an.

Der Begriff «Video Game Essays» beinhaltet zunächst drei zentrale Aspekte, die auf den Gesamtkomplex verweisen:

Der Verwendung des Begriff «Essay» ist zunächst nicht eindeutig und wird insbesondere im allgemeinen Sprachgebrauch in vielfältiger Weise genutzt. Nach dem Duden ist ein Essay (der oder das ist als Artikel möglich) eine Abhandlung, die eine literarische oder wissenschaftliche Frage in knapper und anspruchsvoller Form behandelt. Allerdings sind umfangreichere Essays gerade im wissenschaftlichen Bereich gebräuchlich. Die englische Verwendung der Bezeichnung "Essay» gilt in der Mitte des 20. Jahrhunderts «für Texte, die unsystematisch Betrachtungen über die vielfältigsten Aspekte des menschlichen Lebens wiedergeben. Die Anstrengungen, das Wort der Sache nach adäquat ins Deutsche zu übertragen, sind vielfältig und führen zu Ausdrücken wie ‘Versuch〉, 〈Entwurf〉, 〈Abhandlung〉, 〈Aufsatz〉, ¿Fragment` bzw. zu einer ganzen Reihe von metaphorischen Umschreibungen wie 〈Abschweifung〉, ‘Gespräch», 〈Bemühung〉, ‘Gedanken», ¿Experiment», ‘Annäherung〉, 〈Spiels, <intellektuelle Reise〉 etc.» (Pfammater 2002, S. 7) Eine Definition des Begriffs bzw. eine Eingrenzung erscheint allgemein als schwierig (vgl. Rohner 1966 und Pfammater 2002). Dies liegt u. a. auch an der inhaltlichen Ungebundenheit des

2 Wie komplex und aufwändig eine Bildinterpretation schon allein eines Fotos sein kann, wird deutlich, wenn man erziehungswissenschaftliche Literatur zu diesem Thema sichtet (vgl. Marotzki/Niesyto 2006 und Ehrenspeck 2003). 
Essays: «Das Thema des Essays ist grundsätzlich frei» (Rohner 1966, S. 360). Dies bedeutet, dass sich Autoren in dieser Ausdrucksform mit jeglichen Aspekten eines Themas beschäftigen können, das gerade in ihrem Interesse steht. Das Essay soll in diesem Kontext als eine Form der intensiven Auseinandersetzung mit einem selbst gewählten Thema verstanden werden, das durch die anderen beiden im Begriff enthaltenen Aspekte «Video» und «Video Game» noch genauer spezifiziert und eingegrenzt wird.

Zu dieser näheren Bestimmung der Form dient der zentrale Aspekt «Video», der die Darstellung des Essays in filmischer Form impliziert. Dies bedeutet, dass keine schriftliche Analyse vorgelegt wird, sondern ein filmisches Produkt als «Unterform» des dokumentarischen Films, das die Auseinandersetzung mit dem Thema in spezifischer Form präsentiert. Der Vorteil liegt in der Möglichkeit symbolische Ausdrucksformen der Bildsprache nicht in verbale Abstraktionen zu übersetzen, sondern diese im Kontext des Spiels und seiner Visualisierung zu belassen und mit verbalen Kommentaren zu ergänzen. Hierzu werden für die Analyse relevante Sequenzen des Spiels mit einem Screenrecorderprogramm aufgenommen, in ein Videoschnittprogramm importiert und mit einer verbalen Kommentierung in eine erzählende und zugleich erklärende Form zusammengefügt. Ein Beispiel soll dies verdeutlichen:

Bei dem Spiel «Die Siedler» ${ }^{3}$ sollen der Wirtschaftskreislauf, die Interdependenzen der einzelnen Manufakturen und damit verbunden der Berufe aufgezeigt werden. So wäre es möglich, in einem ersten Schritt bei den basalen Strukturen anzufangen, um dem Betrachter des Essays in das Spiel einzuführen. Um Gebäude bauen zu können, benötigt man Rohstoffe wie Holz und Stein sowie Arbeiter zum Aufbau, die wiederum mit Werkzeugen ausgestattet sind. Somit ist es zunächst notwendig mit den vorhandenen Ressourcen Holzfällerhütten und Sägewerke zu bauen. Durch einen Förster wird der vollständige Kahlschlag des Waldes durch Neuanpflanzungen kleiner Bäume vermieden. Der Steinmetz sorgt in Gebieten mit entsprechenden Vorkommen von Stein für das zweite Baumaterial. Mit dieser grundlegenden Versorgung können dann weitere Zweige wie Nahrungsproduktion, Bergwerke und Metallverarbeitung etc. aufgebaut werden. Hier bietet es sich z. B. an, den Produktionsablauf an der Ressourcengewinnung und -verarbeitung analog eines Dokumentarfilms aufzuzeigen und zu kommentieren, also vom Fällen des Baumes, über die Verarbeitung im Sägewerk und der schlussendlichen Verwendung beim Aufbau neuer Gebäude. Dieses kurze Beispiel soll nur verdeutlichen, wie die Vorgehensweise und Bearbeitung der Produktion des filmischen Essays aussehen kann. Die Komplexität hängt ganz von der Betrachtungsweise und den Interessen des «Essayisten» ab.

Die Beschreibung des Wirtschaftskreislaufs als Ausschnitt aus dem Gesamtkomplex ist noch einfach zu beschreiben. Dies wird umso schwieriger, je symbolhafter die Darstellung der Details wird und man zum Verständnis auf diese zurückgreifen muss. 
Über den letzten Begriffkomplex «Video Game» wird der Gegenstandsbereich des Essays festgelegt. Thematisch stehen hierbei Digital Games (das beinhaltet sowohl Computer- als auch Konsolenspiele) im Blickpunkt der Betrachtung. Um welche Art Spiel es sich handelt, ob Lernspiel oder Edutainmentsoftware, bleibt dabei unerheblich. Dieser begriffliche Vorsatz dient der thematischen Eingrenzung und vor allem der für den Rezipienten erkenntlichen Verortung, wie dies z. B. bei «politischen» oder "geschichtlichen» Essays der Fall ist. In diesem Punkt unterscheidet sich die Arbeit an einem Video Game Essay sowohl von der klassischen Video- und Filmarbeit als auch der Erstellung eines Machinima-Films (vgl. Kelland/ Morris/Lloyd 2005). Das zentrale Thema bildet das Computerspiel, das in der heutigen Lebenswelt der Jugendlichen wie einleitend beschrieben seinen festen Platz hat und so der Forderung nachkommt, mit der aktiven Medienarbeit an «aktuelle Themen bzw. Gegenstände, an denen Jugendliche ihr Interesse bekunden und/ oder die sie als Problem artikulieren» (Schell 2003, S. 149), anzuknüpfen.

Zusammengefasst und auf den Punkt gebracht sind Video Game Essays intensive, distanziert reflexive Auseinandersetzungen mit Computerspielen, die in filmischer Form präsentiert werden. Video Game Essays versuchen, anderen dokumentarischen Genres vergleichbar, in einer Kombination aus erklärenden und erzählenden Elementen Aspekte von Computer- und Videospielen zu erforschen und in einem filmischen Beitrag darzustellen.

\section{Video Game Essays im Anwendungsfeld medienpädagogischer Arbeit}

Diese Kombination aus klassischer Video- bzw. Filmarbeit und dem Gegenstandsbereich Computerspiele lässt sich durchaus auf klassische Arbeitsfelder der aktiven Medienarbeit, die Hochschulausbildung von Studierenden und die Arbeit in der Schule portieren. Während die aktive Medienarbeit mit Video bzw. Film sich der Kamera und dem Filmschnitt als Mittel zur Gestaltung bedient, ist es bei einem Video Game Essay vor allem der von dem Spiel vorgegebene, durch die Programmierung bedingte Blickwinkel. Dies kann zu Einschränkungen oder zu weiteren Möglichkeiten (z. B. Vogelperspektiven wie in einem Flugzeug) in der Gestaltung führen. Hier sind die Gemeinsamkeiten mit Machinima-Filmen zu sehen: Beide Formen nutzen die Bildschirmausgabe der Computerspielsoftware für die visuelle Darstellung: «lt can [...] be recorded in real-time within the virtual environment, much like filmmaking (the majority of game-specific Machinima pieces are produced in this fashion)» (Academy of Machinima Arts and Science 2005). Mit dem Einsatz von Video Game Essays ist eine Fokussierung auf 3-D-Spiele nicht existent. Alle Arten und Genre von Computerspielen können für eine Auseinandersetzung mit digitalen Spielen im Film verwendet werden, vor allem weil Computerspiele selbst im Blickpunkt stehen und diese nicht ausschliesslich für eine Umsetzung filmischer Inszenierungen genutzt werden. Damit werden die Gemeinsamkeiten und Differenzen von Video Game Essays, dem Drehen eines «klassischen〉 Films und 
der Produktion von Machinima deutlich. Während die Filmproduktion als Grundlage zur Gestaltung der anderen beiden gelten kann, schränken Video Game Essyas die Thematik auf Computerspiele ein und öffnen zugleich die Fokussierung der Machinima auf 3-D-Spiele. Damit sind Video Game Essays eine Auseinandersetzung mit einem Medium über ein Medium, was Lernprozesse in vielfältiger Weise zulässt.

Im Gegensatz zum Game Based Learning steht jedoch weniger die Wissensvermittlung selbst im Vordergrund sondern wesentlich stärker die Förderung von Kompetenzen (z. B. Medienkompetenz, Methodenkompetenz etc.). Dies bedeutet jedoch nicht, dass sich diese beiden Bereiche ausschliessen. Die drei genannten Bereiche Medienarbeit, Hochschule und Schule nehmen auf Grund differenter Rahmenbedingungen Einfluss auf die Einsatzmöglichkeiten der filmischen Essays. Daraus ergeben sich unterscheidbare Methoden und Ziele, die mit solchen Projekten Verknüpft sind. Im Folgenden werden diese drei Bereiche im Hinblick auf die Anforderungen, Einsatzmöglichkeiten und Lernziele dargestellt, um den offenen Charakter und die Potentiale der Video Game Essays vorzuführen.

\section{Video Game Essays in der aktiven Medienarbeit}

Das Hauptziel der aktiven Medienarbeit ${ }^{4}$ ist die Förderung von Medienkompetenz $z^{5}$ über ein spezifisches methodisches Vorgehen, welches sich bei der klassischen Videoarbeit etabliert und bewährt hat. Die dafür notwendige technische Ausrüstung für den digitalen Videoschnitt findet sich in nahezu jedem Medienzentrum in ausreichender Qualität und entsprechend den aktuellen Standards. Für die Produktion von Video Game Essays sind allerdings einige weitere Punkte zu beachten: So muss z. B. berücksichtigt werden, dass die Rechner die Mindestanforderungen der Spiele an die Hardware übertreffen, da eine zusätzliche Software (Screenrecorder) benötigt wird, die parallel zum Spiel aktiv sein muss und das Spielgeschehen, welches auf dem Bildschirm zu sehen ist, als Videodatei auf der Festplatte speichert. Weiterhin muss die entsprechende Spielesoftware vorhanden und für das Alter der Teilnehmer freigegeben sein.

Daraus ergibt sich die Frage, welche spezifischen Lernziele bei der Produktion von Video Game Essays im spezifischen Anwendungsrahmen in den Blickpunkt rücken und welche methodischen Grundannahmen der aktiven Medienarbeit dabei Berücksichtigung finden: Während Teilnehmer von Medienprojekten bei der Videoarbeit den Inhalt der Videos möglichst frei bestimmen können und so ihre individuellen Interessen und Probleme als Thema aufgreifen können, grenzt der Fokus «Video Games» dies zunächst ein. Da Computerspiele - wie einleitend be-

\footnotetext{
4 Auf die Kriterien aktiver Medienarbeit wird im weiteren Verlauf immer wieder Bezug genommen, ohne diese dabei in breiter Form auszuführen und zu definieren. Für eine intensive Auseinandersetzung siehe Schell (2003), dessen Ausführungen als Grundlage herangezogen wurden.

5 Als Basis für die weiteren Ausführungen wird die Dimensionierung nach Baacke (1999) in die Bereiche Medienkritik, Mediennutzung, Mediengestaltung und Medienkunde zu Grunde gelegt.
} 
schrieben - im Alltag von Heranwachsenden ihren festen Platz eingenommen haben, dürften sich auch hier ohne Probleme Themen finden lassen, die dem Ziel Rechnung tragen, «dass medienpädagogisches Handeln an der individuellen Ausgangslage der Jugendlichen zu orientieren ist» (Schell 2003, S. 52). So wäre es zum Beispiel möglich, dass Jugendliche sich darauf einigen in Gruppenarbeit - als ein zentrales Element der aktiven Medienarbeit - ein Essay zu produzieren, das an ihre Eltern gerichtet ist und über Inhalte, Ziele sowie die Faszination des Spiels Auskunft geben. Um dies zu bewerkstelligen, müssen Jugendliche sich verschiedener Aspekte bewusst werden: Sie müssen zum Beispiel über das Spiel selbst und dessen spezifische Strukturen, die Faszination, die davon ausgeht, und das eigene anzufertigende Produkt inkl. der Arbeitsschritte der Herstellung reflektieren. Wichtig für die Reflexion ist dabei, dass sie jederzeit auf die Unterstützung der Medienpädagogen zurückgreifen können und dieser neue Impulse in die Gruppe trägt. Der gesamte Prozess kann in unterschiedliche Phasen eingeteilt werden: Im ersten Schritt werden die verinnerlichten Spielstrukturen erkundet und wieder ins Bewusstsein zurückgeführt, da die Jugendlichen diese durch das vertraute Spielen zunächst verinnerlicht haben und unbewusst geworden sind. D. h. die habituellen Muster werden aus der Ebene der unbewussten Kompetenz in die Ebene der bewussten Kompetenz transferiert (vgl. Mrochen 2001, S. 18). Hierbei lernen die Jugendlichen Spiele zu analysieren, deren Machart zu bestimmen und auf ihre eigenen Vorlieben kritisch zu übertragen. Dabei ist es durchaus möglich auf vorhandene Erfahrungen aus der Medienarbeit zurückgreifen. Mit einem vorbereiteten Fragenkatalog können Auseinandersetzungen mit dem ausgewählten Spiel initiiert werden (vgl. Müller 2001, S. 49-50):

- Wie gefällt dir das Spiel?

- Was interessiert oder langweilt dich an diesem Spiel?

- Wie beurteilst du das Verhalten der Spielfiguren?

- Hat das Spiel etwas mit dir zu tun?

Diese Punkte könnten im Verlauf des Projekts z. B. als Kapitelstruktur für das Essay dienen, was den Jugendlichen als Leitfaden zur Auseinandersetzung mit dem Computerspiel helfen kann. Komplexer kann dies gestaltet werden, wenn die Jugendlichen selbst einen Kriterienkatalog erarbeiten, welche Aspekte von Computerspielen bei dem Essay Berücksichtigung finden sollen. Grafik, Steuerung, Motivationsförderung, Spielspass und Rahmengeschichte können beispielsweise als Kriterien Verwendung finden. Hilfreich zur Einführung in die Thematik können bei der Verwendung eines Kriterienkatalogs gängige auf dem Markt erhältliche Spielezeitschriften sein, die Bewertungen von neuen Spielen auf den beiliegenden Datenträgern in Form von Videos bereithalten. Der Grad der Komplexität eines Video-Game-Essay-Projekts lässt sich so auf die Zielgruppe anpassen, damit eine Über- oder Unterforderung vermieden wird. 
Nach der Phase der grundlegenden Auseinandersetzung mit dem Spiel geht es darum, dies in die filmische Form des Essays zu übertragen. Anhand eines «Drehbuchs» legen die Teilnehmer fest, welche Narrations- und Argumentationsstruktur ihr Essay haben soll. Hierbei wird auch der Vorteil der filmischen Form der Auseinandersetzung ersichtlich: «In der Arbeit am stehenden oder bewegten Bild lassen sich Spiele auch dann deskriptiv erfassen, wenn die Schriftform als Beschreibungsmedium dem Entwicklungsstand der Spieler nicht angemessen ist» (Müller 2001, S. 50) oder die ‘Übersetzung) der Bildinformationen in die Schriftform als unangemessen erscheint. Das Konzept der Video Game Essays stellt eine Möglichkeit dar, Computerspiele in audiovisueller Weise zu erfassen, zu analysieren und vor allem mit einer präsentativen Symbolik (vgl. Niesyto 2003, S. 17) angemessen darzustellen. Niesyto rekurriert in seinen Ausführungen auf Langer (1987/1942), nach der «Menschen ihre Lebenserfahrungen und ihre Gefühle in symbolischer Form» (Niesyto 2003, S. 17) verarbeiten. Hierbei ist die diskursive Sprache nicht die einzige Form, da auch Bilder, Musik, Tanz, Körpersprache usw. als präsentative Symbolik ihre - nicht zu geringe - Bedeutung haben (vgl. Niesyto 2003, S. 17). Genau hier können individuelle Spielerfahrungen von Jugendlichen bearbeitet und präsentiert werden. Die filmische Form der Auseinandersetzung mit Computerspielen bietet die Möglichkeiten, sich sowohl mit Sprache in Form der Moderation und über die grafisch symbolischen Darstellung der Spiele auszudrücken. Dabei ist nicht ausgeschlossen, dass die Jugendlichen eine andere präsentative Symbolik z.B. aus einem Film einfügen, um sich mit dem Spiel auseinanderzusetzen. Dies bietet sich gerade mit zunehmenden Tendenzen medienkonvergenter Inhalte an. Nach Abschluss der Produktion der Essays ist es durchaus sinnvoll, diese auch einem Publikum zu zeigen. Ob dies über eine Präsentation geschieht oder im Internet als Videostream erfolgt, hängt vor allem von den urheberrechtlichen Fragen ab. Manche Anbieter von Videospielen sehen solche Videos durchaus als Werbung an und tolerieren eine öffentliche Verbreitung.

Zusammengefasst stehen folgende (Lern-)Ziele im Fokus der Anwendung der Video Game Essays in der freien Medienarbeit:

- Reflektieren über Computerspiele und sich über deren individuelle Bedeutung (persönlicher Bezug) bewusst werden

- Sozialkompetenzen (z. B. durch Gruppenarbeit, Diskussionen)

- Kenntnisse über die Handhabung und Bedienung der Hard- und Software

- Kenntnisse über die Möglichkeiten der Mediengestaltung

\section{Video Game Essays in der Hochschulausbildung}

Das zweite Einsatzgebiet, das in exemplarischer Kurzform (als Erfahrungsbericht) dargestellt wird, ist die Ausbildung im Bachelor-Studiengang Medienbildung an der Universität Magdeburg, in dem Computerspiele als Wahlpflichtfach fest in die Studien- und Prüfungsordnung verankert wurden. Im Rahmen eines Seminars wur- 
de das Thema Video Game Essays aufgegriffen, diskutiert und praktisch umgesetzt. Dabei wurde basierend auf bereits vermittelten und grundlegenden Kenntnissen zu Computerspielen gefragt, wie diese adäquat zu einer Produktion eines Video Game Essays in der Medienarbeit mit Jugendlichen eingesetzt werden können. Das bereits oben beschriebene Szenario kann dabei als Zielvorstellung dienen. Dazu wurden die Studierenden im Seminar mit den Fragen «Was könnte ein Video Game Essay sein?», «Wie plane ich didaktisch im Sinne der aktiven Medienarbeit ein Video-Game-Essay-Projekt?», «Welche Ziele werden damit verfolgt?» und «Wie produziert man ein filmisches Essay über Computerspiele (technische Durchführung)?» konfrontiert.

Zu Beginn des Seminars wurden die Studierenden aufgefordert, anhand von Beispielvideos und ihrem bisherigen Wissen einen Kriterienkatalog aufzustellen, welche Themen ein Video Game Essay aufgreifen kann. Mit Hilfe dieses Katalogs wurde die Bedeutung der einzelnen Kriterien für bestimmte Zielgruppen diskutiert. So entstand ein zweiter erweiterter und auf verschiedene Zielgruppen (Eltern, Lehrer/ innen, Jugendliche) abgestimmter Katalog. Hierdurch konnten die Studierenden auf theoretische und praktische Erfahrungen der zielgruppenorientierten Medienarbeit zurückgreifen und diese auf neue Arbeitsfelder beziehen. Daran anschliessend wurden von erfahrenen Studierenden die Kenntnisse über die konkrete technische Umsetzung der Aufnahme von Spielsequenzen, der Videoschnitt, die Vertonung und die Ausgabe der Essays in Form von internetfähigen Videostreams an die anderen Seminarteilnehmer vermittelt. Mit diesen technischen Kompetenzen ausgestattet wurden alle Teilnehmer in kleine Gruppen eingeteilt, um eigenständig ein Essay zu produzieren. Sie konnten dabei frei wählen, welches Spiel sie thematisieren und welche Zielgruppenperspektive sie einnehmen wollten. So mussten die Gruppen jeweils das Spiel analysieren, dessen mögliche Bedeutung für die Zielgruppe diskutieren und in ihr Essay einbringen. Zuletzt wurden die Filmessays den anderen Seminarteilnehmern präsentiert und zur Diskussion gestellt.

Zusammengefasst sind folgende (Lern-)Ziele in der Hochschullehre bei der Nutzung von Video Game Essays zu konstatieren:

- Vermittlung und Anwendung grundlegender Kenntnisse über Computerspiele und ihre Verortung in vornehmlich kindlichen und jugendlichen Lebenswelten (Zielgruppenorientierung)

- Didaktische Planung von Projekten und deren Durchführung (Methodenkompetenz)

- Kenntnisse über die Handhabung und Bedienung der notwendigen Hard- und Software

- Förderung medienpädagogischer Kompetenzen (vgl. Blömecke 2000)

- Kenntnisse über die Möglichkeiten der Mediengestaltung

- Sozialkompetenzen (z. B. durch Gruppenarbeit, Diskussionen) 


\section{Video Game Essays in der Schule}

Mittlerweile ist in den Lehrplänen die Förderung von Medienkompetenz verankert, was den Einsatz der Video Game Essays in der Schule fächerübergreifend ermöglicht. Problematisch ist hier der Mangel an Zeit für umfangreiche Projekte und die Hardwareausstattung der Schulen, die als eher unzureichend für die ressourcenhungrigen Computerspiele angesehen werden kann (vgl. die Rechnertypen aus der Studie des BMBF 2005, S. 8). Von daher ist der Einsatz in der Schule lediglich möglich, wenn der Umfang der Video Game Essays geringer gehalten wird oder sie im Rahmen einer Projektwoche zum Einsatz kommen. Die Auswahl der Spiele begrenzt sich dabei auf ältere Spiele, die weniger Rechenleistung und spezielle Hardware (wie 3-D-Grafikkarten) benötigen.

Wie der Einsatz einer verkürzten Form gestaltet werden kann, soll ein Beispiel aufzeigen. Im Geschichtsunterricht wie auch in etlichen Computerspielen wird das Mittelalter als Thema aufgegriffen. Hier bietet es sich an, die visuellen Elemente der Spiele mit realen zeitgeschichtlichen Gegenständen und Gebäuden zu vergleichen, um einerseits Wissen über die historischen Fakten zu vermitteln und andererseits - wenn vorhanden ${ }^{6}$ - die medialen Freiheiten der Mediengestalter und die Abweichungen zu den Fakten zu verdeutlichen. Ein Essay würde somit Computerspiele dazu nutzen, klassische Wissensbestände aus dem Lehrplan zu vermitteln und Medienkompetenz (insbesondere die Dimension der Medienkritik) zu fördern. Diese Vorgehensweise bietet den Vorteil, Schülerinteressen (Computerspiele, Medieninhalte) mit der klassischen Wissensvermittlung zu verbinden. Der entscheidende Nachteil ist darin zu sehen, dass die Form der Auseinandersetzung - mit Ausnahme von Projektwochen - nur in Ansätzen dem Prozesscharakter gerecht werden kann, der dem Konzept zu Grunde liegt.

Zusammengefasst existieren folgende (Lern-)Ziele in der Schule beim Einsatz von Video Game Essays :

- Unterstützung der Wissensvermittlung bei den im Lehrplan verankerten Inhalten

- Förderung von Medienkompetenz (Medienkritik und Medienkunde)

\section{Fazit}

Video Game Essays bieten über die ursprüngliche Idee einer wissenschaftlichen Analyseform von Computerspielen hinaus Möglichkeiten, um Lernprozesse bei Jugendlichen, Studierenden und Schülern anzuregen. Im Gegensatz zu klassischen Lernspielen stehen nicht die durch das Spiel initiierten Prozesse zur Wissensvermittlung im Blickpunkt, sondern jene, die sich um das Spiel herum ergeben. Die Digital Games dienen hier als Initialzündung für weitergehende Prozesse, die

So wurde beim Spiel Assassin's Creed versucht, die historischen Städte Jerusalem und Damaskus möglichst genau nachzubilden. 
durch Medienpädagogen/innen und Lehrpersonen an Schulen und Hochschulen durch die Gestaltung der "Lernsettings» angeregt und angeleitet werden. Den Spielen kommt damit eine Bedeutung zu, die sich zwischen Unterhaltungs- und Lernmedium ansiedeln lässt. Dies bietet den Vorteil, dass man auf Spiele zurückgreifen kann, an denen die Jugendlichen Interesse haben und die ihnen vertraut sind. Ein Spagat wie bei Lernspielen, «langweilige» Lerninhalte möglichst unterhaltsam an den Vorlieben der Jugendlichen orientiert in eine Spielgeschichte zu verpacken und auf gleiches Interesse wie bei Entertainment-Software zu hoffen, ist dabei nicht notwendig und auch nicht gewollt.

Je nach Einsatzzweck der Video Game Essays stehen teilweise unterschiedliche Prozesse und Ziele im Vordergrund: Bei der Jugendarbeit ist die Förderung von Medienkompetenz und Sozialkompetenz ein vorrangiges Anliegen, während in der Hochschulausbildung auf bereits erworbene basale Kenntnisse über Computerspiele zur Ausbildung von Methodenkompetenz für die Gestaltung einer didaktischen bzw. projektorientierten Lernsituation zurückgegriffen wird. In diesem Kontext werden diese Kenntnisse zudem erweitert und ausgebaut. In der Schule dienen Video Game Essays durchaus stärker dem «klassischen» Ziel der Wissensvermittlung, indem sie unterstützend in den schulischen Lernprozess integriert werden. Und genau hier zeigen sich auch die Grenzen einer solchen spezifischen Form der medialen Auseinandersetzung: Thematisch müssen Überschneidungspunkte zwischen den verwendeten Computerspielen, der filmischen Form der Video Game Essays und den Zielvorstellungen existieren. Prozess, Inhalt und Ziel eines Projekts müssen aufeinander abgestimmt werden. Die prinzipiell offene Form der Video Game Essays schafft einen möglichen Spielraum bei den Einsatzgebieten, schränkt diesen aber zugleich auch ein, indem technische Besonderheiten und die filmische Auseinandersetzung mit Computerspielen zu berücksichtigen sind.

Die Vorteile des Einsatzes überwiegen jedoch. Gerade in Verbindung mit den Kriterien der aktiven Medienarbeit (vgl. Schell 2003) lassen sich vielfältige (Lern-) Prozesse initiieren, gerade auch dann, wenn es darum geht, die unterschiedlichen Grundvoraussetzungen (Lebensweltbezug, Kompetenzförderung, institutioneller Kontext, unterschiedliche und variabel festsetzbare Schwerpunkte der visuellen oder verbalen Kommunikation) zu verbinden.

\section{Literatur}

Aarseth, Espen (2001): Computer Game Studies, Year One. In: Game Studies. The international journal of computer game research. Vol. 1, issue 1, July 2001. Online unter http://www.gamestudies.org/0101/editorial.html, zuletzt geprüft am 7.8.2008.

Academy of Machinima Arts and Science (2005): the machinima faq. Online unter http://www.machinima.org/machinima-faq.html, zuletzt besucht am 14.11.2008 
Baacke, Dieter (1999): Medienkompetenz als zentrales Operationsfeld von Projekten. In: Baacke, Dieter; Kornblum Susanne; Lauffer, Jürgen; Mikos, Lothar; Thiele, Günter A. (Hrsg.): Handbuch Medien: Medienkompetenz. Modelle und Projekte. Bonn: Bundeszentrale für politische Bildung. S. 31-35.

Blömeke, Sigrid (2000): Medienpädagogische Kompetenz. Theoretische und empirische Fundierung eines zentralen Elements der Lehrerausbildung. München: kopaed.

Bundesministerium für Bildung und Forschung (BMBF) (Hrsg.) (2005): IT-Ausstattung der allgemein bildenden und berufsbildenden Schulen in Deutschland. Bestandsaufnahme 2005 und Analyse 2001 bis 2005. Bonn: Bundesministerium für Bildung und Forschung (BMBF) Referat Öffentlichkeitsarbeit (BMBF publik).

Bundesverband Interaktive Unterhaltungssoftware e.V.: http://www.biu-online.de/ fileadmin/user/dateien/BIU___Marktzahlen_1._Habljahr_2007.pdf, zuletzt geprüft am 11.02.2008.

Egenfeldt-Nielsen, Simon; Smith, Jonas Heide; Tosca, Susana Pajares (2008): Understanding video games. The essential introduction. New York, NY: Routledge.

Ehrenspeck, Yvonne. (2003): Film- und Fotoanalyse in der Erziehungswissenschaft. Ein Handbuch. Opladen: Leske + Budrich.

Fritz, Jürgen (1995): Modelle und Hypothesen zur Faszinationskraft von Bildschirmspielen. In: Fritz, Jürgen (Hrsg.): Warum Computerspiele faszinieren. Empirische Annäherungen an Nutzung und Wirkung von Bildschirmspielen. Weinheim: Juventa. S. 11-38.

Fritz, Jürgen (1997): Zur «Landschaft» der Computerspiele. In: Fritz, Jürgen; Fehr, Wolfgang (Hrsg.): Handbuch Medien: Computerspiele. Bonn: Bundeszentrale für politische Bildung. S. 87-97.

Fromme, Johannes; Meder, Norbert (Hrsg.) (2001): Bildung und Computerspiele. Zum kreativen Umgang mit elektronischen Bildschirmspielen. Opladen: Leske + Budrich.

Gebel, Christa; Gurt, Michael; Wagner, Ulrike (2005): Kompetenzförderliche Potenziale populärer Computerspiele. In: Arbeitsgemeinschaft BeTRIEBliche Weiterbildungsforschung e.V. (Hrsg.): E-Lernen: Hybride Lernformen, OnlineCommunities, Spiele. QUEM-report, Heft 92. Berlin , S. 241-376.

Kelland, Matt; Morris, Dave; Lloyd, Dave (2005): Machinima. Making animated movies in 3D virtual environments. Lewes: Ilex.

Kommer, Sven/Biermann, Ralf (2007): Zwischen Erinnerung und Inszenierung - Medienbiografien medial. In: Freiburger FrauenStudien «Erinnern und Geschlecht, Band II», Band 20, S. 195-220.

Kraam, Nadia (2004): Kompetenzfördernde Aspekte von Computerspielen. In: merz I medien + erziehung, Heft 3, S. 12-17. 
Langer, Susanne (1987/1942): Philosophie auf neuem Wege. Frankfurt/Main: Fischer.

Löschenkohl, Erich (1995): Faszination Computerspiel. Eine psychologische Bewertung. 1. Aufl. Wien: ÖBV Pädag. Verl.

Marotzki, Winfried; Niesyto, Horst (Hrsg.) (2006): Bildinterpretation und Bildverstehen. Methodische Ansätze aus sozialwissenschaftlicher, kunst- und medienpädagogischer Perspektive. Medienbildung und Gesellschaft, 2. Wiesbaden: VS Verlag für Sozialwissenschaften.

Marotzki, Winfried; Jörissen, Benjamin (2008): Wissen, Artikulation und Biographie: Theoretische Aspekte einer Strukturalen Medienbildung. In: Fromme, Johannes; Sesink, Werner (Hrsg.): Pädagogische Medientheorie. Wiesbaden: VS Verlag für Sozialwissenschaften, S. 51-70.

Medienpädagogischer Forschungsverbund Südwest (2007): KIM-Studie 2006 (Kinder und Medien, Computer und Internet). Basisuntersuchung zum Medienumgang 6- bis 13-Jähriger. Stuttgart.

Medienpädagogischer Forschungsverbund Südwest (2006): JIM-Studie 2006 (Jugend, Information, (Multi-)Media). Basisuntersuchung zum Medienumgang 12- bis 19-Jähriger. Stuttgart: Medienpädagogischer Forschungsverbund Südwest.

Müller, Kai (2001): Computerspiele reflektieren - Einsatzmöglichkeiten von «Search\&Play». In: Fromme, Johannes; Meder, Norbert (Hrsg.): Bildung und Computerspiele. Zum kreativen Umgang mit elektronischen Bildschirmspielen. Opladen: Leske + Budrich, S. 43-56.

Mrochen, Siegfried (2001): Kompetenzen - was ist das eigentlich? In: Zeitschrift Siegen:Sozial (SI:SO). Heft 1, S. 16-18.

Neitzel, Britta; Bopp, Matthias; Nohr, Rolf F. (Hrsg.) (2005): «See? I'm real...». Multidisziplinäre Zugänge zum Computerspiel am Beispiel von «Silent Hillı. 2., unveränd. Aufl. Münster: LIT Verlag.

Niesyto, Horst (2003): VideoCulture. Projektentwicklung und Projektergebnisse. In: Niesyto, Horst (Hrsg.): VideoCulture. Video und interkulturelle Kommunikation [Grundlagen, Methoden und Ergebnisse eines internationalen Forschungsprojekts]. München: kopaed. S. 15-110.

Pfammatter, René (2002): Essay - Anspruch und Möglichkeit. Plädoyer für die Erkenntniskraft einer unwissenschaftlichen Darstellungsform. Schriftenreihe Poetica, 67. Hamburg: Kovac.

Rohner, Ludwig (1966): Der deutsche Essay. Materialien zur Geschichte und Ästhetik einer literarischen Gattung. Neuwied: Luchterhand.

Schell, Fred (2003): Aktive Medienarbeit mit Jugendlichen. Theorie und Praxis. 4. unveränderte Aufl. Reihe Medienpädagogik, Bd. 5. München: kopaed. 